\title{
Saneamento básico em domicílios indígenas de áreas urbanas da Amazônia Legal, Brasil
}

\section{Basic sanitation in indigenous households in urban areas of Brazil's Legal Amazon}

\author{
Gerson Luiz Marinho (D), Ludimila Raupp² (D), José Rodolfo Mendonça de Lucena ${ }^{3}$ (D), \\ Felipe Guimarães Tavares ${ }^{4}$ (D) \\ 'Departamento de Enfermagem de Saúde Pública, Escola de Enfermagem Anna Nery, Universidade Federal do Rio de Janeiro - \\ Rio de Janeiro (RJ), Brasil. \\ ${ }^{2}$ Departamento de Biologia, Pontifícia Universidade Católica do Rio de Janeiro - Rio de Janeiro (RJ), Brasil. \\ ${ }^{3}$ Escola Nacional de Saúde Pública, Fundação Oswaldo Cruz - Rio de Janeiro (RJ), Brasil. \\ ${ }^{4}$ Escola de Enfermagem Aurora de Afonso Costa, Universidade Federal Fluminense - Niteroi (RJ), Brasil.
}

Como citar: Marinho GL, Raupp L, Lucena JRM, Tavares FG. Saneamento básico em domicílios indígenas de áreas urbanas da Amazônia Legal, Brasil. Cad Saúde Colet, 2021;29(esp.):177-186. https://doi.org/10.1590/1414-462X202199010455

\section{Resumo}

Introdução: Os indígenas apresentam expressivas vulnerabilidades socioeconômicas e epidemiológicas em comparação aos não indígenas. Quase metade da população indígena residente em áreas urbanas estava na Amazônia Legal em 2010 (46,8\%). Objetivo: Analisar a infraestrutura relacionada ao saneamento básico de domicílios urbanos dentro e fora da Amazônia Legal, com foco nos indígenas. Método: Artigo descritivo sobre as condições de saneamento básico dos domicílios com responsáveis indígenas e não indígenas, realizado a partir de dados do Censo Demográfico 2010. Foram calculadas as frequências para abastecimento de água, esgotamento sanitário e destino do lixo segundo a localização na Amazônia Legal. As magnitudes das ocorrências foram analisadas por meio de razões de prevalência. Resultados: No Brasil, 114 mil domicílios urbanos eram indígenas, dos quais 17,4\% eram localizados na Amazônia Legal. Na região, 6 em cada 10 domicílios não possuíam tratamento de esgoto adequado. Os domicílios indígenas apresentaram chances mais elevadas de ter saneamento básico precário. Na Amazônia, a chance de domicílios indígenas não possuírem instalações sanitárias adequadas foi o dobro daquela observada para os demais domicílios. Conclusão: Mesmo em situações em que os contingentes populacionais eram mais expressivos, os indígenas apresentavam notórias desigualdades em relação ao restante da população.

Palavras-chave: saneamento básico; iniquidade em saúde; população indígena; censos.

\begin{abstract}
Background: In Brazil, the indigenous population presents the highest socioeconomic and epidemiological vulnerabilities. In $2010,46.8 \%$ of the urban indigenous population lived in the Legal Amazon socio-geographic division. Objective: To analyze the basic sanitation infrastructure of urban dwellings inside and outside the Legal Amazon with a focus on indigenous people. Method: Descriptive study on the basic sanitation conditions of households whose heads were indigenous and non-indigenous individuals according to the 2010 Brazilian Census. Frequencies were calculated for water supply, sanitary sewage, and waste destination according to location in the Legal Amazon. Prevalence ratios were calculated to compare the groups. Results: In 2010, there were 114,600 urban indigenous domiciles in Brazil, and $17.4 \%$ of them were located in the Legal Amazon. Six out of 10 urban indigenous domiciles did not have adequate sewage treatment. Indigenous domiciles had higher chances of having precarious basic sanitation. In the Legal Amazon, indigenous domiciles presented a 2-fold chance of not having
\end{abstract}

Este é um artigo publicado em acesso aberto (Open Access) sob a licença Creative Commons Attribution, que permite uso, distribuição e reprodução em qualquer meio, sem restrições desde que o trabalho original seja corretamente citado.
Trabalho realizado a partir de análises de dados censitários (Censo Demográfico 2010 - IBGE).

Correspondência: Gerson Luiz Marinho. E-mail: marinho@eean.ufrj.br

Fonte de financiamento: nenhuma.

Conflito de interesses: nada a declarar.

Recebido em: Set. 21, 2019. Aprovado em: Dez. 26, 2019 
adequate sanitation facilities compared with that of other domiciles. Conclusion: Even in situations where the population groups are more expressive, indigenous people present important inequalities in relation to the rest of the population.

Keywords: basic sanitation; health status disparities; indigenous population; censuses.

\section{INTRODUÇÃO}

A construção de indicadores sociodemográficos e de saúde a partir de fontes nacionalmente representativas permite o reconhecimento das necessidades da população e auxilia no delineamento e no desenvolvimento de políticas públicas equitativas ${ }^{1}$. As desigualdades e as iniquidades em saúde são reconhecidamente influenciadas pelas condições socioeconômicas e apresentam diferentes magnitudes quando consideram aspectos relativos aos diversos pertencimentos étnico-raciais referidos pela população ${ }^{2-4}$.

Entre os indicadores que descrevem as condições de vida, aqueles que se referem às condições da infraestrutura relacionada ao saneamento básico refletem, direta e indiretamente, o modo como se expressam os problemas ligados à saúde pública e às perdas nas áreas social e econômica ${ }^{5}$. A presença e a qualidade desses serviços têm influência direta sobre o perfil de morbimortalidade da população, desempenho e atraso escolar das crianças, absenteísmo e produtividade entre os trabalhadores, valorização de imóveis, turismo, desenvolvimento econômico local e regional, além da conservação dos meios físico e biótico ${ }^{5-8}$.

No Brasil, são mais de 35 milhões de pessoas sem acesso aos serviços relacionados ao saneamento básico 5 . Em áreas urbanas, onde residem cerca de $90 \%$ da população, essa questão assume relevância ainda mais expressiva ${ }^{5,8}$. Apesar dos avanços ligados à presença de infraestrutura sanitária registrados nos últimos anos ${ }^{9}$, eles não foram suficientes para garantir o acesso universal, integral e de qualidade para a totalidade dos domicílios localizados dentro do território nacional. No tocante às desigualdades regionais, as cidades localizadas no Norte e Nordeste do Brasil permanecem com os mais elevados déficits de acesso a serviços de saneamento ${ }^{5,9,10}$.

$\mathrm{Na}$ linha das desigualdades socioeconômicas, as condições de saúde e saneamento mais deficitárias se somam às desigualdades regionais e expõem aos segmentos classificados como"preto e pardo" as maiores vulnerabilidades ambientais $2,4,10$. Os estudos sobre a realidade das condições de saneamento entre os indígenas ainda são escassos, sendo a maior parte restrita a contextos locais do Brasil, como o norte de Minas Gerais ${ }^{11}$ e o noroeste da região amazônica $^{12}$. Mais recentemente, a partir de dados nacionalmente representativos e foco nas macrorregiões, foi constatado que os indígenas residentes em áreas urbanas apresentam condições de infraestrutura de saneamento básico expressivamente inferiores em relação às demais categorias de cor ou raça ${ }^{13}$.

De acordo com o Censo Demográfico 2010, 12,8\% do total da população brasileira residia em municípios que compõem a região denominada Amazônia Legal, que compreende todos os estados da região Norte e parte dos estados do Mato Grosso (Centro-Oeste) e Maranhão (Nordeste) ${ }^{14}$. Por outro lado, do total de indígenas, $46,8 \%$ residiam nessa região, o que sinaliza para a relevância do desenvolvimento de estudos específicos para esse contexto geográfico. Assim, a descrição da infraestrutura sanitária domiciliar considerando segmentos populacionais com recortes étnico-raciais e localização geográfica é relevante para fomentar debates acerca de ações e políticas públicas direcionadas a segmentos populacionais minoritários.

Nesse sentido, o objetivo deste estudo foi analisar a presença de infraestrutura dos serviços relacionados ao saneamento básico entre os domicílios urbanos localizados dentro e fora da Amazônia Legal, com foco nos indígenas, a partir do Censo Demográfico 2010.

\section{MÉTODO}

O estudo analisou os dados do Censo Demográfico 2010 realizado pelo Instituto Brasileiro de Geografia e Estatística (IBGE), coletados por meio do questionário básico aplicado a todos os domicílios existentes no território brasileiro (universo) no período da coleta dos dados ( $1^{\circ}$ de agosto a 31 de outubro de 2010). Os dados foram acessados no Banco Multidimensional 
de Estatísticas (BME) (www.bme.ibge.gov.br), ferramenta que divulga dados agregados das pesquisas nacionais realizadas pelo Instituto. As análises foram realizadas a partir da seleção dos domicílios urbanos localizados dentro e fora dos limites da Amazônia Legal.

De acordo com o IBGE, "áreas urbanas" eram aquelas que, urbanizadas ou não, estavam localizadas no perímetro urbano das cidades ou vilas, além das áreas urbanas isoladas, segundo estabelecido legalmente em cada município na data de 31 de julho de $2010^{15}$. A região geográfica denominada "Amazônia Legal" abrange uma área de 5.217 .423 km², o que corresponde a $60 \%$ do território brasileiro e 775 municípios, sendo todos da região Norte e parte dos estados do Maranhão e Mato Grosso. A Amazônia Legal foi instituída para fins de planejamento econômico da região amazônica por meio da Lei Complementar n 124 , de 3 de janeiro de $2007^{16}$.

"Domicílio indígena" foi definido como aquele cujo morador responsável foi classificado na categoria "indígena" na pergunta sobre cor ou raça, ao passo que o domicílio "não indígena" correspondeu ao conjunto das demais categorias: "branca", "preta", "amarela" e "parda". Para fins da coleta dos dados do Censo Demográfico 2010, a "pessoa responsável pelo domicílio" foi identificada por meio do quesito "condição no domicílio" [código V0502], definida como "a pessoa (homem ou mulher), com no mínimo 10 (dez) anos de idade, reconhecida pelos moradores como responsável pelo domicílio"15 (p. 317).

Os responsáveis indígenas e não indígenas dos domicílios localizados dentro e fora da Amazônia Legal foram caracterizados segundo aspectos demográficos e socioeconômicos (sexo, idade, alfabetização e rendimento nominal mensal). As perguntas sobre alfabetização e renda incluíram pessoas com pelo menos 5 e 10 anos, respectivamente. Na época da coleta de dados do Censo 2010, o salário mínimo era igual a $\mathrm{R} \$$ 510,00 (data de referência: 31 de julho de 2010) ${ }^{15}$.

As características domiciliares referentes à infraestrutura de saneamento básico foram descritas a partir de três variáveis investigadas pelo questionário básico do Censo 2010, quais sejam: "esgotamento sanitário" [código = V0204], "abastecimento de água" [V0205] e "destino do lixo" [V0206] (Quadro 1).

Quadro 1. Variáveis que caracterizam as condições de saneamento básico nos domicílios e critérios de adequação dos serviços. Censo Demográfico 2010

\begin{tabular}{|c|c|c|}
\hline Variável - código & Adequado & Não adequado \\
\hline \multirow{4}{*}{ Esgotamento sanitário - V0204 } & 1. Rede geral de esgoto ou pluvial & 3. Fossa rudimentar \\
\hline & \multirow{3}{*}{ 2. Fossa séptica } & 4. Vala \\
\hline & & 5. Rio, lago ou mar \\
\hline & & 6. Outro \\
\hline \multirow{9}{*}{ Abastecimento de água - V0205 } & \multirow{9}{*}{ 1. Rede geral de distribuição } & 2. Poço ou nascente na propriedade \\
\hline & & $\begin{array}{l}\text { 3. Poço ou nascente fora da } \\
\text { propriedade }\end{array}$ \\
\hline & & 4. Carro-pipa \\
\hline & & $\begin{array}{l}\text { 5. Água da chuva armazenada em } \\
\text { cisterna }\end{array}$ \\
\hline & & $\begin{array}{l}\text { 6. Água da chuva armazenada de outra } \\
\text { forma }\end{array}$ \\
\hline & & 7. Rios, açudes, lagos e igarapés \\
\hline & & 8. Outra \\
\hline & & 9. Poço ou nascente na aldeia (indígena)* \\
\hline & & $\begin{array}{l}\text { 10. Poço ou nascente fora da aldeia } \\
\text { (indigena)* }^{*}\end{array}$ \\
\hline \multirow{5}{*}{ Destino do lixo - V0206 } & $\begin{array}{l}\text { 1. Coletado diretamente por serviço de } \\
\text { limpeza }\end{array}$ & 3. Queimado (na propriedade) \\
\hline & \multirow{4}{*}{$\begin{array}{l}\text { 2. Colocado em caçamba de serviço de } \\
\text { limpeza }\end{array}$} & 4. Enterrado (na propriedade) \\
\hline & & $\begin{array}{l}\text { 5. Jogado em terreno baldio ou } \\
\text { logradouro }\end{array}$ \\
\hline & & 6. Jogado em rio, lago ou mar \\
\hline & & 7. Tem outro destino \\
\hline
\end{tabular}

*Somente para domicílios em terras indígenas 
Os critérios de adequação dos domicílios quanto à oferta e disponibilidade de instalações sanitárias básicas utilizados pelo IBGE por ocasião da investigação censitária foram amparados pela Lei no 11.445/07, principal marco regulatório de saneamento básico no Brasil ${ }^{17}$. Assim, foram considerados adequados aqueles domicílios com água proveniente da rede geral de abastecimento, escoadouros ligados à rede geral ou fossa séptica e lixo coletado direta ou indiretamente pelos serviços de limpeza (Quadro 1).

Os resultados foram apresentados em tabelas descritivas que comparam as frequências de domicílios indígenas e não indígenas em relação às características relacionadas aos serviços de saneamento básico dentro e fora da Amazônia Legal. As magnitudes da presença de infraestrutura que caracterizam o saneamento básico nos domicílios foram mensuradas por meio de odds ratio (OR), que estima as chances de os domicílios com saneamento "não adequado" terem responsáveis indígenas.

Quanto à ética em pesquisa, os resultados do Censo Demográfico 2010 são disponibilizados pelo IBGE para acesso público. De acordo com a legislação vigente, por serem dados secundários de acesso irrestrito (Resolução n 466/2012, do Conselho Nacional de Saúde), não houve necessidade de aprovação do Comitê de Ética em Pesquisa.

\section{RESULTADOS}

De acordo com o Censo Demográfico 2010, havia aproximadamente 58 milhões de domicílios em todo Brasil, sendo a maior parte em áreas urbanas (85,7\%). Do total de domicílios indígenas urbanos no país, 19.829 (17,3\%) estavam localizados em municípios da Amazônia Legal, representando quase o dobro do contingente dos domicílios não indígenas situados na região (Tabela 1).

Tabela 1. Distribuição dos domicílios indígenas e não indígenas segundo a localização dentro e fora da Amazônia Legal, áreas urbanas, Brasil, 2010

\begin{tabular}{ccccccc}
$\begin{array}{c}\text { Amazônia } \\
\text { Legal }\end{array}$ & Indígenas & $\%$ & $\begin{array}{c}\text { Não } \\
\text { indígenas }\end{array}$ & $\%$ & Total & $\%$ \\
\hline Sim & 19.829 & 17,3 & 4.675 .200 & 9,5 & 4.695 .029 & 9,5 \\
\hline Não & 94.771 & 82,7 & 44.519 .729 & 90,5 & 44.614 .500 & 90,5 \\
\hline Total & $\mathbf{1 1 4 . 6 0 0}$ & $\mathbf{1 0 0 , 0}$ & $\mathbf{4 9 . 1 9 4 . 9 2 9}$ & $\mathbf{1 0 0 , 0}$ & $\mathbf{4 9 . 3 0 9 . 5 2 9}$ & $\mathbf{1 0 0 , 0}$ \\
\hline
\end{tabular}

Fonte: Censo Demográfico 2010

Os responsáveis pelos domicílios indígenas eram majoritariamente do sexo feminino em ambos os contextos geográficos (dentro e fora da Amazônia Legal). As idades dos responsáveis não apresentaram variações importantes na comparação entre indígenas e não indígenas (Tabela 2).

Responsáveis pelos domicílios urbanos que não sabiam ler e escrever foram mais frequentes entre os indígenas na Amazônia Legal, assim como em relação ao grupo de pessoas que não possuíam rendimentos. $O$ volume de domicílios com responsáveis indígenas sem rendimento em municípios da Amazônia Legal foi aproximadamente o dobro dos domicílios não indígenas localizados fora da Amazônia Legal, com valores de 20,7\% e 11,8\%, respectivamente (Tabela 2).

A não adequação dos domicílios em relação à infraestrutura de saneamento básico foi mais frequente naqueles em que os responsáveis eram indígenas em ambos os contextos (Figura 1 e Tabela 3).

A magnitude das frequências de domicílios com condições não adequadas de saneamento na Amazônia Legal indica a precariedade desses serviços tanto para indígenas quanto para não indígenas, com marcante situação da ligação à rede geral de esgotamento sanitário. A pior frequência relacionada aos serviços de saneamento básico se revelou entre domicílios urbanos 
Tabela 2. Características demográficas e socioeconômicas dos responsáveis indígenas e não indígenas de domicílios localizados dentro e fora da Amazônia Legal, áreas urbanas, Brasil, 2010

\begin{tabular}{|c|c|c|c|c|}
\hline & \multicolumn{2}{|c|}{ Amazônia Legal } & \multicolumn{2}{|c|}{ Fora da Amazônia Legal } \\
\hline & Indígenas & Não indígenas & Indígenas & Não indígenas \\
\hline & $N=19.829$ & $N=4.675 .200$ & $N=94.771$ & $N=44.519 .729$ \\
\hline & $\%$ & $\%$ & $\%$ & $\%$ \\
\hline \multicolumn{5}{|l|}{ Sexo } \\
\hline Masculino & 54,5 & 57,7 & 54,8 & 59,2 \\
\hline Feminino & 45,5 & 42,3 & 45,2 & 40,8 \\
\hline \multicolumn{5}{|l|}{ Idade } \\
\hline De 10 a 19 anos & 2,6 & 1,9 & 1,9 & 1,3 \\
\hline De 20 a 29 anos & 16,7 & 17,8 & 14,6 & 13,5 \\
\hline De 30 a 39 anos & 23,8 & 25,6 & 21,8 & 22,2 \\
\hline De 40 a 49 anos & 21,7 & 22,2 & 21,9 & 22,5 \\
\hline De 50 a 59 anos & 16,5 & 15,8 & 18,7 & 18,5 \\
\hline 60 anos ou mais & 18,7 & 16,7 & 21,1 & 22,0 \\
\hline \multicolumn{5}{|l|}{ Alfabetizados } \\
\hline Sim & 82,9 & 87,9 & 87,1 & 91,1 \\
\hline Não & 17,1 & 12,1 & 12,9 & 8,9 \\
\hline \multicolumn{5}{|c|}{ Rendimento nominal mensal } \\
\hline Sem rendimento & 20,7 & 14,8 & 13,5 & 11,8 \\
\hline Até $1 \mathrm{SM}^{*}$ & 42,8 & 37,6 & 36,8 & 28,0 \\
\hline Mais de 1 a $2 \mathrm{SM}$ & 19,9 & 23,4 & 26,4 & 26,9 \\
\hline Mais de 2 a 5 SM & 11,8 & 15,9 & 16,9 & 21,5 \\
\hline Mais de $5 \mathrm{SM}$ & 4,7 & 8,2 & 6,5 & 11,8 \\
\hline
\end{tabular}

*SM = salário mínimo; equivalente a R\$ 510,00 à época da coleta de dados do Censo. Fonte: Censo Demográfico (2010)

com responsáveis indígenas na Amazônia Legal, onde 66,4\% não possuíam esgoto ligado à rede geral (Figura 1 e Tabela 3).

As chances de serviços básicos de saneamento não adequado foram significativamente mais elevadas para os domicílios indígenas, em ambos os contextos analisados (dentro e fora da Amazônia Legal) (Tabela 3). Chama atenção a magnitude da diferença do abastecimento de água em domicílios indígenas na Amazônia Legal, onde foi observado que a chance de um domicílio indígena não ser abastecido com água tratada foi o dobro daquela registrada para os demais domicílios ( $\mathrm{OR}=2,37 ; p<0,01)$ (Tabela 3 ).

\section{DISCUSSÃO}

O direito ao saneamento básico é reconhecido pela Constituição Federal e assegurado pela Lei Federal $n^{\circ} 11.445 / 2007$, que preconiza sua universalização e integralidade da oferta ${ }^{17}$. O dispositivo também define as ações de saneamento básico como o conjunto de serviços de infraestrutura operacional de abastecimento de água potável em quantidade e qualidade 


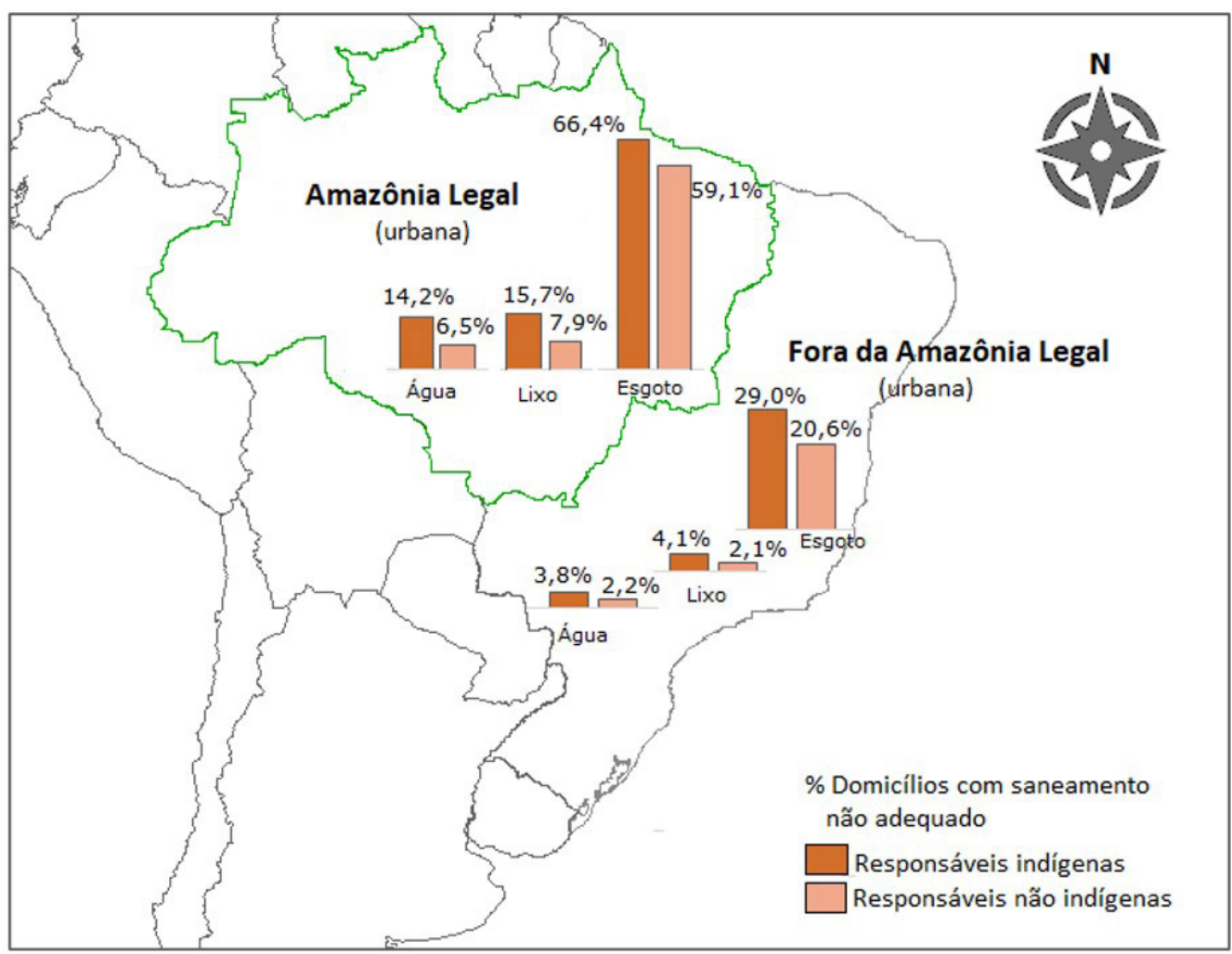

Figura 1. Domicílios indígenas e não indígenas com condições não adequadas de saneamento. Amazônia Legal, áreas urbanas, Brasil, 2010. Nota: condições "não adequadas" se referem à ausência de abastecimento de água por rede geral, ausência de esgoto por rede geral e de fossa séptica e ausência de coleta de lixo por serviço de limpeza. Fonte: Censo Demográfico 2010

Tabela 3. Características de saneamento básico dos domicílios indígenas e não indígenas localizados dentro e fora da Amazônia Legal, áreas urbanas, Brasil, 2010

\begin{tabular}{|c|c|c|c|c|c|}
\hline \multicolumn{3}{|c|}{ Amazônia Legal } & \multicolumn{3}{|c|}{ Fora da Amazônia Legal } \\
\hline Indígenas & $\begin{array}{c}\text { Não } \\
\text { indígenas }\end{array}$ & & Indígenas & $\begin{array}{c}\text { Não } \\
\text { indígenas }\end{array}$ & \\
\hline $\begin{array}{c}n \\
=19.707\end{array}$ & $\begin{array}{c}n= \\
4.661 .985\end{array}$ & OR & $\begin{array}{c}n= \\
94.301\end{array}$ & $\begin{array}{c}n= \\
44.449 .881\end{array}$ & OR \\
\hline (\%) & (\%) & & (\%) & (\%) & \\
\hline 85,8 & 93,5 & $2,37^{*}$ & 96,2 & 97,8 & $1,80^{*}$ \\
\hline 14,2 & 6,5 & & 3,8 & 2,2 & \\
\hline
\end{tabular}

\begin{tabular}{|c|c|c|c|c|c|c|}
\hline \multicolumn{7}{|c|}{ Abastecimento de água } \\
\hline Adequado & 85,8 & 93,5 & $2,37^{*}$ & 96,2 & 97,8 & $1,80^{*}$ \\
\hline Não adequado & 14,2 & 6,5 & & 3,8 & 2,2 & \\
\hline \multicolumn{7}{|c|}{ Esgotamento sanitário } \\
\hline Adequado & 33,6 & 40,9 & $1,36^{*}$ & 71,0 & 79,4 & $1,57^{*}$ \\
\hline Não adequado & 66,4 & 59,1 & & 29,0 & 20,6 & \\
\hline \multicolumn{7}{|l|}{ Destino do lixo } \\
\hline Adequado & 84,3 & 92,1 & $2,16^{*}$ & 95,9 & 97,9 & $2,03^{*}$ \\
\hline Não adequado & 15,7 & 7,9 & & 4,1 & 2,1 & \\
\hline
\end{tabular}

${ }^{*} p<0,01$. Fonte: Censo Demográfico (2010)

$\mathrm{OR}=$ Odds Ratio 
adequada, coleta, tratamento e destinação final adequados do esgotamento sanitário e resíduos sólidos e drenagem das águas pluviais ${ }^{17}$.

No âmbito internacional, o Brasil é país signatário do protocolo que ratifica o Direito Humano à Água e ao Esgotamento Sanitário, sem discriminação e para toda população, reconhecendo essas questões como diretamente relacionadas às boas condições de vida ${ }^{18,19}$. Por sua condição de país-membro da Organização das Nações Unidas (ONU), também deve investir em compromissos políticos que fomentem o alcance dos Objetivos de Desenvolvimento Sustentável, estratégia que, por meio de 17 objetivos, propõe-se, entre outras questões, a erradicar a pobreza, assegurar uma vida saudável e promover o bem-estar de todos. Entre os objetivos, os países devem assegurar e garantir a disponibilidade e gestão sustentável da água e saneamento para todos, reduzir as desigualdades e promover maior justiça social ${ }^{19}$.

Alinhados aos objetivos estabelecidos como metas para o alcance de melhores condições de vida, os países-membros da ONU também se comprometem a reconhecer, por meio de instrumentos utilizados em inquéritos nacionais, a diversidade étnica e racial que neles residem, incluindo os povos indígenas ${ }^{20,21}$. Seguindo tais recomendações, o reconhecimento da diversidade racial no Brasil vem sendo tradicionalmente realizado por intermédio de categorias que determinam dimensões relacionadas à "cor ou raça" e estão presentes não apenas nos recenseamentos populacionais, mas em vários sistemas de informações governamentais, inclusive no âmbito da saúde ${ }^{3,14}$.

Os censos populacionais vêm sendo realizados com periodicidade decenal há mais de dois séculos, e o IBGE é o órgão estatal responsável pela condução dos recenseamentos desde a edição de $1940^{3}$, em geral realizados decenalmente desde $1872^{14}$. O quesito referente à classificação étnico-racial no país experimentou uma série de alterações no decorrer da história dos censos. Atualmente, a pergunta é "qual sua cor ou raça", tendo como categorias de resposta as opções "branca, "preta", "amarela", "parda" e "indígena"14.

Enquanto proxy das condições de vida e saúde, os estudos demográficos e epidemiológicos mantêm a tradição de considerar a população brasileira estratificada segundo dois grupos raciais, quais sejam:"brancos" e"negros", quase sempre excluindo os segmentos minoritários, como os "amarelos" e os"indígenas" partir de dados censitários, nacionalmente representativos) apontam sistematicamente maiores desvantagens para os indígenas em comparação ao restante da população ${ }^{13,22}$.

É crescente o número de estudos que descrevem a vulnerabilidade entre os indígenas no Brasil diante da precariedade do perfil epidemiológico desse segmento ${ }^{2,4,11-13,22-27}$. Os achados aqui descritos coadunam com o conjunto dos resultados da literatura referida e reiteram as hipóteses de desigualdades e iniquidades, com destaque para o segmento indígena da população brasileira, o que se verifica, inclusive, em relação às condições de saneamento dos domicílios ${ }^{13}$.

A partir de diferentes fontes de dados, sejam eles oriundos dos sistemas de informação em saúde ou das pesquisas domiciliares nacionais, a exemplo dos censos demográficos, as desigualdades étnico-raciais se expressam de modo contundente, indicando maior vulnerabilidade socioeconômica e de saúde para os indígenas. O perfil de saúde da população indígena, em particular do segmento infantil, está estreitamente relacionado com a precariedade da infraestrutura de saneamento básico dos domicílios ${ }^{28,29}$.

Realizado em terras indígenas, o Inquérito de Saúde e Nutrição dos Povos Indígenas revelou que uma em cada quatro crianças tinha baixo peso para altura (indicador sensível para déficit de crescimento por consequências nutricionais) e mais da metade apresentava anemia $(51,2 \%)^{30}$. Além disso, aproximadamente um quarto das crianças tinham sido internadas por diarreia na semana anterior à entrevista ${ }^{30}$. Essas prevalências se mostraram associadas às características de saneamento dos domicílios. De maneira geral, os indicadores epidemiológicos menos satisfatórios foram encontrados na região Norte, em domicílios de estratos socioeconômicos mais baixos e com piores condições sanitárias, coincidindo em grande parte com a abrangência da Amazônia Legal|26,27,29,30. 
Apesar de não existirem indicadores epidemiológicos representativos para os indígenas residentes em áreas urbanas, as fontes de dados demográficos, em especial os censos, vêm demonstrando que a presença de pessoas que se reconhecem como indígenas é cada vez mais expressiva em contextos urbanos. De acordo com o Censo 2010, aproximadamente $40 \%$ dos quase 900 mil indígenas identificados no Brasil moravam nas cidades ${ }^{14}$.

As análises apresentadas neste estudo indicam que, nas áreas urbanas, os domicílios indígenas se encontram em desvantagem em relação aos não indígenas e que as desvantagens já descritas para diversos indicadores de vida e saúde se reproduzem para as condições de infraestrutura de saneamento básico no Brasil.

O estudo das condições de vida a partir de dados nacionalmente representativos são úteis no diálogo com políticas públicas voltadas à redução de desigualdades. No caso da população indígena, as vantagens podem ser mencionadas em relação aos resultados estratificados segundo a situação dos domicílios (urbana e rural), além do potencial de serem verificadas informações em diversos contextos espaciais, entre os quais a região denominada Amazônia Legal.

Apesar de ter sido criada com objetivo principal de monitoramento das condições de preservação e manejo das reservas florestais, a Amazônia Legal é uma região com expressiva presença de populações indígenas, que vêm se apresentando com volumes cada vez maiores nas cidades da região. Ao que parece, ainda que presentes em contingentes mais significativos comparativamente às regiões Sudeste e Sul, os indígenas das cidades amazônicas estão submetidos a expressivas vulnerabilidades, incluindo condições de moradia.

A presença dos indígenas nas cidades sinaliza para a realização de estudos mais detalhados, uma vez que os dados censitários demonstram cenários de sistemática desigualdade em comparação ao restante da população.

\section{REFERÊNCIAS}

1. Cunha JMP. Demografia e políticas públicas: uma combinação sugestiva e necessária. In: Marques E, Faria CAP, editores. A política como campo multidisciplinar. 2. ed. São Paulo: Editora UNESP; Rio de Janeiro: Editora Fiocruz; 2018. p. 207-30. http://dx.doi.org/10.7476/9786557080825.0010.

2. Comissão Nacional sobre Determinantes Sociais da Saúde. As causas sociais das iniquidades em saúde no Brasil. Rio de Janeiro: Editora Fiocruz; 2008.

3. Henriques R. Desigualdade racial no Brasil: evolução das condições de vida na década de 90. Rio de Janeiro: Instituto de Pesquisa Econômica Aplicada; 2001.

4. Chor D. Desigualdades em saúde no Brasil: é preciso ter raça. Cad Saude Publica. 2013;29(7):1272-5. http://dx.doi.org/10.1590/S0102-311X2013000700002. PMid:23842995.

5. Instituto Trata Brasil. Benefícios econômicos e sociais da expansão do saneamento no Brasil [Internet]. São Paulo:Trata Brasil; 2018 [citado em 2019 jan 18]. Disponível em: http://tratabrasil.org.br/estudos/ estudos-itb/beneficios-economicos-e-sociais

6. Teixeira JC, Gomes MHR, Souza JA. Associação entre cobertura por serviços de saneamento e indicadores epidemiológicos nos países da América Latina: estudo com dados secundários. Rev Panam Salud Publica. 2012;32(6):419-25. http://dx.doi.org/10.1590/S1020-49892012001400005. PMid:23370185.

7. Soares SRA, Bernardes RS, Cordeiro OM No. Relações entre saneamento, saúde pública e meio ambiente: elementos para formulação de um modelo de planejamento em saneamento. Cad Saude Publica. 2002;18(6):1713-24. http://dx.doi.org/10.1590/S0102-311X2002000600026. PMid:12488899.

8. Ayach LR, Guimarães S, Cappi N, Ayach C. Saúde, saneamento e percepção de riscos ambientais urbanos [Internet]. Cad Geogr. 2012;22(37):47-64 [citado em 2019 jan 19]. Disponível em: http:// periodicos.pucminas.br/index.php/geografia/article/view/3021/3865

9. Borja PC. Política pública de saneamento básico: uma análise da recente experiência brasileira. Saude Soc. 2014;23(2):432-47. http://dx.doi.org/10.1590/S0104-12902014000200007.

10. Instituto Brasileiro de Geografia e Estatística. Coordenação de População e Indicadores Sociais. Síntese de indicadores sociais: uma análise das condições de vida da população brasileira. Rio de Janeiro: IBGE; 2018. 
11. Pena JL, Heller L. Saneamento e saúde indígena: uma avaliação na população Xakriabá, Minas Gerais. Eng Sanit Ambient. 2008;13(1):63-72. http://dx.doi.org/10.1590/S1413-41522008000100009.

12. Toledo RF, Giatti LL, Pelicioni MCF. Mobilização social em saúde e saneamento em processo de pesquisa-ação em uma comunidade indígena no noroeste amazônico. Saude Soc. 2012;21 (1):206-18. http://dx.doi.org/10.1590/S0104-12902012000100020.

13. Raupp L, Fávaro TR, Cunha GM, Santos RV. Condições de saneamento e desigualdades de cor/raça no Brasil urbano: uma análise com foco na população indígena com base no Censo Demográfico de 2010. Rev Bras Epidemiol. 2017;20(1):1-15. http://dx.doi.org/10.1590/1980-5497201700010001. PMid:28513790.

14. Instituto Brasileiro de Geografia e Estatística. Os indígenas no Censo Demográfico 2010: primeiras considerações com base no quesito cor ou raça. Rio de Janeiro: IBGE; 2012.

15. Instituto Brasileiro de Geografia e Estatística. Metodologia do Censo Demográfico 2010. Rio de Janeiro: IBGE; 2013.

16. Brasil. Lei Complementar n. ${ }^{\circ} 124$ de 3 de janeiro de 2007. Institui, na forma do art. 43 da Constituição Federal, a Superintendência do Desenvolvimento da Amazônia - SUDAM. Diário Oficial da União, 4 de janeiro de 2007.

17. Brasil. Lei n. ${ }^{\circ} 11.445$, de 5 de janeiro de 2007. Estabelece diretrizes nacionais para o saneamento básico. Diário Oficial da União, 8 de janeiro de 2007.

18. United Nations General Assembly. Human right to water and sanitation. Geneva: UNGA; 2010. (UN Document; no. A/RES/64/292).

19. United Nations General Assembly. Transforming our world: the 2030 Agenda for Sustainable Development. New York: UN; 2015. 35 p. (Report N A/RES/70/1).

20. United Nations. Principles and recommendations for population and housing censuses. Revision 2 . New York: UN; 2008.

21. United Nations. State of the world's indigenous peoples. New York: UN; 2009.

22. Bastos JL, Santos RV, Cruz OG, Longo LAFB, Silva LO. Características sociodemográficas de indígenas nos censos brasileiros de 2000 e 2010: uma abordagem comparativa. Cad Saude Publica. 2017;33(Supl. 1):e00085516. http://dx.doi.org/10.1590/0102-311x00085516.

23. Campos MB, Borges GM, Queiroz BL, Santos RV. Diferenciais de mortalidade entre indígenas e não indígenas no Brasil com base no Censo Demográfico de 2010. Cad Saude Publica. 2017;33(5):e00015017. http://dx.doi.org/10.1590/10.1590/0102-311x00015017. PMid:28614445.

24. Caldas ADR, Santos RV, Borges GM, Valente JG, Portela MC, Marinho GL. Mortalidade infantil segundo cor ou raça com base no Censo Demográfico de 2010 e nos sistemas nacionais de informação em saúde no Brasil. Cad Saude Publica. 2017;33(7):e00046516. http://dx.doi.org/10.1590/0102-311x00046516. PMid:28792987.

25. Marinho GL, Caldas ADR, Santos RV. Indigenous residents in "improvised" households according to the 2010 brazilian national census. Physis. 2017;27(1):79-102.

26. Horta BL, Santos RV, Welch JR, Cardoso AM, dos Santos JV, Assis AM, et al. Nutritional status of indigenous children: findings from the First National Survey of Indigenous People's Health and Nutrition in Brazil. Int J Equity Health. 2013;12(1):23. http://dx.doi.org/10.1186/1475-9276-12-23. PMid:23552397.

27. Leite MS, Cardoso AM, Coimbra CE Jr, Welch JR, Gugelmin SA, Lira PC, et al. Prevalence of anemia and associated factors among indigenous children in Brazil: results from the First National Survey of Indigenous People's Health and Nutrition. Nutr J. 2013;12(1):69. http://dx.doi.org/10.1186/14752891-12-69. PMid:23714275.

28. Neves-Silva P, Heller L. O direito humano à água e ao esgotamento sanitário como instrumento para promoção da saúde de populações vulneráveis. Cien Saude Colet. 2016;21(6):1861-70. http://dx.doi. org/10.1590/1413-81232015216.03422016. PMid:27281669.

29. Escobar AL, Coimbra CE Jr, Welch JR, Horta BL, Santos RV, Cardoso AM. Diarrhea and health inequity among Indigenous children in Brazil: results from the First National Survey of Indigenous People's Health and Nutrition. BMC Public Health. 2015;15(1):191. http://dx.doi.org/10.1186/s12889-0151534-7. PMid:25880758. 
30. Coimbra CE Jr, Santos RV, Welch JR, Cardoso AM, Souza MC, Garnelo L, et al. The First National Survey of Indigenous People's Health and Nutrition in Brazil: rationale, methodology, and overview results. BMC Public Health. 2013;13(1):52. http://dx.doi.org/10.1186/1471-2458-13-52. PMid:23331985. 